\title{
Cinq récepteurs pour la dopamine : mais où s'arrêteront-ils ?
}

Jusqu'à il y a quelques mois, seulement deux récepteurs dopaminergiques avaient été caractérisés. Il s'agissait des récepteurs $D_{1}$ et $D_{2}$, couplés respectivement de manière positive et négative à l'adénylate cyclase. On savait également que le récepteur $\mathrm{D}_{2}$ était la cible commune de tous les neuroleptiques, dont les propriétés antipsychotiques sont utilisées pour soigner certaines affections psychiatriques comme la schizophrénie.

Pourtant, des observations aussi bien pharmacologiques que comportementales [1] laissaient supposer depuis longtemps une multiplicité des sites d'action des neuroleptiques, sans qu'une preuve définitive soit apportée.

La situation a commencé à changer en 1988 lorsque le groupe d'Olivier Civelli a effectué le clonage de l'ADNc du récepteur dopaminergique $\mathrm{D}_{2}$ [2]. En utilisant des sondes nucléotidiques dérivées de la séquence du récepteur $\mathrm{D}_{2}$, plusieurs groupes, dont le nôtre [3], avaient, dans un premier temps, mis en évidence l'existence de deux isoformes du récepteur $\mathrm{D}_{2}$ ayant le même profil pharmacologique, mais dont le rôle physiologique n'est pas encore déterminé.

Puis, les choses se sont précipitées avec, tout d'abord, le clonage par quatre équipes différentes des gènes du récepteur $D_{1}$ [4-7], suivi de celui du récepteur dopaminergique $\mathrm{D}_{3}$, en premier lieu chez le rat [8] puis chez l'homme [9], qui défaisait le dogme de l'existence de seulement deux récepteurs pour la dopamine. Enfin, c'est aujourd'hui le clonage des gènes de deux nouveaux récepteurs dopaminergiques, $D_{4}$ et $D_{5}[10,11]$ qui porte le nombre de récepteurs dopaminergiques à au moins cinq. Ces cinq récepteurs dopaminergiques sont $\mathrm{m} / \mathrm{s} n^{\circ} 6$, vol. 7, juin-juillet 91 tous construits sur le modèle de la rhodopsine avec sept domaines transmembranaires et un couplage avec une protéine $\mathrm{G}$.

Leur caractérisation moléculaire (Tableau I) permet donc d'avoir des informations sur leur localisation cérébrale et périphérique, leur pharmacologie (Tableau II) et leur voie de transduction, et d'envisager leur importance et leur rôle relatif dans les systèmes de transmission dopaminergique.

Récepteurs dopaminergiques $D_{1}$ et $D_{5}$. Le gène du récepteur $D_{5}$ a été cloné dans une banque génomique humaine à l'aide d'un fragment de restriction du récepteur $\mathrm{D}_{1}[10]$. Ce nouveau récepteur est très proche du récepteur $\mathrm{D}_{1}$, avec une homologie de $50 \%$, alors qu'elle n'est que de $30 \%$ avec les récepteurs $\mathrm{D}_{2}$ et $\mathrm{D}_{3}$. Comme le récepteur $D_{1}$, le récepteur $\mathrm{D}_{5}$ est transcrit à partir d'un gène sans intron, et lorsqu'il est exprimé dans des cellules eucaryotes appropriées (GH4-C1), son activation provoque la stimulation de l'adénylate cyclase. Son ARNm est contenu dans la plupart des grandes régions dopaminergiques (tubercule olfactif, bulbe olfactif, caude-putamen, accumbens) à l'exclusion de l'hypophyse et de la glande parathyroïde qui constitue la localisation prototype du récepteur $D_{1}$. Son profil pharmacologique est quasiment le même que celui du récepteur $D_{1}$, avec pourtant une différence importante, puisqu'en effet la dopamine, qui est l'agoniste endogène, semble le reconnaître dix fois mieux.

Récepteurs dopaminergiques $D_{2}$, $\mathrm{D}_{3}$ et $\mathrm{D}_{4}$. L'ADNc du récepteur $\mathrm{D}_{4}$ a été cloné à partir d'une banque d'ADNc de neuroblastome humain (SK-N-MC) en utilisant un fragment de restriction du gène du récepteur $\mathrm{D}_{2}$ de rat [11]. Le clone ainsi obtenu étant un clone partiel (de TM4 à la partie C terminale), le reste du gène a été cloné dans une banque génomique humaine. Le gène contient quatre introns, dont trois sont localisés aux mêmes endroits que ceux des gènes des récepteurs $\mathrm{D}_{2}$ et $\mathrm{D}_{3}$ [12] qui en contiennent, respectivement, six et cinq. Son homologie globale avec les récepteurs $\mathrm{D}_{1}, \mathrm{D}_{2}$ et $\mathrm{D}_{3}$ est de respectivement $28 \%, 41 \%$ et $39 \%$. L'analyse par Northern blot dans le cerveau de singe permet de le visualiser dans le striatum, le cortex frontal, le mésencéphale, l'amygdale, la moelle épinière, et à un degré moindre, dans les tubercules olfactifs et l'hippocampe. Son expression semble avoir posé quelques problèmes, étant donnée l'impossibilité d'obtenir un ADNc complet, aussi bien dans une banque d'ADNc que par amplification d'ARNm en utilisant la technique de PCR. Les auteurs ont donc dû faire une construction en fusionnant une partie du gène avec l'ADNc obtenu dans la banque des neuroblastomes, donnant lieu à un ADNc hybride de 3,9 kb qui contient les deux premiers introns. Pour la pharmacologie des agonistes, on observe un déplacement de la liaison de dopamine par un analogue non dégradable du GTP (ce qui démontre indirectement un couplage avec une protéine $\mathrm{G}$ ) avec une affinité de $450 \mathrm{nM}$, soit entre les affinités pour les récepteurs $D_{2}$ et $D_{3}$. En ce qui concerne les antagonistes, pour la plupart des neuroleptiques utilisés en clinique, seuls les halopéridol et le spipérone ont une bonne affinité pour le récepteur $\mathrm{D}_{4}$. En revanche, la chlorpromazine, le sulpiride et le pimozide ont des affinités qui ne semblent pas compatibles avec un 
blocage du récepteur $\mathrm{D}_{4}$ aux doses cliniques habituellement utilisées. La différence la plus marquée est obtenue avec la clozapine, neuroleptique au profil tout à fait particulier qui n'est pratiquement jamais utilisé en première intention, qui a une mauvaise affinité pour les récepteurs $D_{2}$ et $\mathrm{D}_{3}$, alors qu'elle est de $9 \mathrm{nM}$ pour le $\mathrm{D}_{4}$, compatible avec les doses plasmiques mesurées chez des patients qui en reçoivent. Bien que la clozapine ait une affinité encore meilleure pour le récepteur de la sérotonine $5 \mathrm{HT}_{2}(2 \mathrm{nM})$, son action

\begin{tabular}{|l|c|c|c|c|c|}
\hline \multicolumn{7}{|c|}{ Tableau I } \\
\hline \multicolumn{7}{|c|}{ CARACTÉRISTIQUES STRUCTURALES } \\
\hline & $D_{1}$ & $D_{5}$ & $D_{2}$ & $D_{3}$ & $D_{4}$ \\
\hline $\begin{array}{l}\text { Localisation } \\
\text { chromosomique }\end{array}$ & $5 q 31-34$ & ND & $11 q 23-24$ & $3 q 13.3$ & $11 p$ \\
Introns & 0 & 0 & 6 & 5 & 4 \\
Isoformes & ND & ND & $D_{21}$ & $D_{3}$ & ND \\
& & & $D_{2 s}$ & $D_{3}$ (O2-dell & \\
Longueur (a.a.) & 446 & 477 & 443 & 400 & 387 \\
Formation d'AMPc & & & & & $?$ \\
\hline
\end{tabular}

Tableau II

AFFINITÉS ( $n M)$ DES AGONISTES ET ANTAGONISTES, SUR LES RÉCEPTEURS DOPAMINERGIQUES HUMAINS CLONÉS

\begin{tabular}{|c|c|c|c|c|c|}
\hline & $D_{1}$ & $D_{5}$ & $D_{2}$ & $D_{3}$ & $D_{4}$ \\
\hline \multicolumn{6}{|l|}{ Agonistes } \\
\hline $\begin{array}{l}\text { Dopamine + GppNHp } \\
\text { Bromocriptine } \\
\text { Quinpirole } \\
\text { SKF } 38393 \\
\text { Antagonistes }\end{array}$ & $\begin{array}{r}2340 \\
672 \\
>20000 \\
150\end{array}$ & $\begin{array}{r}230 \\
454 \\
>20000 \\
100\end{array}$ & $\begin{array}{rl} & 2000 \\
5,3 & 1400 \\
& 1400 \\
& 5000\end{array}$ & $\begin{array}{r}34 \\
7,4 \\
39 \\
>5000\end{array}$ & $\begin{array}{r}450 \\
340 \\
46 \\
1800\end{array}$ \\
\hline $\begin{array}{l}\text { Halopéridol } \\
\text { Chlorpromazine } \\
\text { (-) Sulpiride } \\
\text { Pimozide } \\
\text { Clozapine } \\
\text { SCH } 23390\end{array}$ & $\begin{array}{r}27 \\
73 \\
36000 \\
-\quad \\
141 \\
0,3\end{array}$ & $\begin{array}{r}48 \\
133 \\
77270 \\
-\quad \\
250 \\
0,3\end{array}$ & $\begin{array}{l}0,6 \\
2,3 \\
10 \\
9,8 \\
69 \\
720\end{array}$ & $\begin{array}{r}2,9 \\
5,9 \\
20 \\
11 \\
479 \\
780\end{array}$ & $\begin{array}{r}5,1 \\
37 \\
52 \\
43 \\
9 \\
3560\end{array}$ \\
\hline
\end{tabular}

sur le récepteur $\mathrm{D}_{4}$ pourrait expliquer ses effets antipsychotiques.

Perspectives. La caractérisation moléculaire d'au moins cinq récepteurs dopaminergiques différents offre des espoirs tout à fait réels pour la compréhension des systèmes dopaminergiques, la recherche de liaisons ou d'associations de polymorphismes de leurs gènes avec certaines maladies psychiatriques, et bien évidemment le développement rationnel d'agonistes et d'antagonistes spécifiques de chacun d'entre eux. tion française a été, ou est actuellement, sous traitement par des neuroleptiques, et que ce traitement est souvent chronique. L'apport le plus immédiat du clonage et de la caractérisation pharmacologique des récepteurs $\mathrm{D}_{2}, \mathrm{D}_{3}$ et $\mathrm{D}_{4}$ est de constater qu'aucun de ces récepteurs à lui seul ne constitue le site unique d'action de tous les neuroleptiques. Nous sommes donc maintenant dans une phase de "pharmacologie inverse", où la cible est caractérisée en premier, et va permettre de développer par des études de relation structureactivité, de nouvelles molécules, véri- tables scalpels chimiques des affections psychiatriques!

Les années à venir vont donc être tout à fait passionnantes, avec l'apparition de ces nouvelles molécules et leur utilisation en clinique qui permettra définitivement de mesurer l'importance des progrès accomplis

\footnotetext{
Bruno Giros

Inserm U. 109, 2ter, nue d'Alésia, 75014 Paris, France.
} 


\section{RÉFÉRENCES}

1. Schwartz JC, Delandre M, Martres MP, et al. Biochemical and behavioral identification of discriminant benzamides derivatives : new tools to differentiate subclasses of dopamine receptors. In : Usdin E, Carlsson A,

Dahlstrom A, Engel J, eds. New York : Alan R. Liss, 1984: 59-72.

2. Bunzow JR, Van Tol HHM, Grandy DK, et al. Cloning and expression of a rat $\mathrm{D}_{2}$ dopamine receptor cDNA. Nature 1988 ; 336 : 783-7.

3. Giros B, Sokoloff P, Martres PM, Riou JF, Emorine LJ, Schwartz JC. Alternative splicing directs the expression of two $\mathrm{D}_{2}$ dopamine receptor isoforms. Nature 1989 ; 342 : 923-6.

4. Dearry A, Gingrich JA, Falardeau P, Fremeau RT, Bates MD, Caron MG. Molecular cloning and expression of the gene for a human $\mathrm{D}_{1}$ dopamine receptor. Nature $1990 ; 347$ : 72-6.

5. Monsma FJ, Mahan LC, McVittie LD, Gerfen CR, Sibley DR. Molecular cloning and expression of a $D_{1}$ dopamine receptor linked to adenylyl cyclase activation. Proc Natl Acad Sci USA 1990 ; 87 : 6723-7.

6. Sunahara RK, Niznik HB, Weiner DM, et al. Human dopamine $D_{1}$ receptor encoded by an intronless gene on chromosome 5. Nature $1990 ; 347$ : 80-3.

7. Zhou QZ, Grandy DK, Thambi L, et al. Cloning and expression of human and rat D dopamine receptor. Nature $1990 ; 347$ : 76-80.

8. Sokoloff P, Giros B, Martres MP, Bouthenet ML, Schwartz JC. Molecular cloning and caracterization of a novel dopamine receptor $\left(D_{3}\right)$ as a target for neuroleptics. Nature 1990; 347 : 146-51.

9. Giros B, Martres MP, Sokoloff P, Schwartz JC. cDNA cloning of the human dopaminergic $\mathrm{D}_{3}$ receptor and chromosome identification. CR Acad Sci 1991; 311 . 501-8.

10. Sunahara RK, Guan HC, O'Dowd BF, et al. Cloning of the gene for a human dopamine $\mathrm{D}_{5}$ receptor with higher affinity for dopamine than $D_{1}$. Nature $1991 ; 350$ : 614-9.

11. Van Tol HHM, Bunzow JR, Guan JC, et al. Cloning of the gene for a human dopamine $\mathrm{D}_{4}$ receptor with high affinity for the antipsychotic clozapine. Nature $1991 ; 350: 610-4$

12. Giros B, Martres MP, Pilon C, Sokoloff P, Schwartz JC. Shorter variants of the $\mathrm{D}_{3}$ dopamine receptor produced through various patterns of alternative splicing. Biochem biophys Res Commun 1991 (sous presse).

\section{TIRÉS A PART}

B. Giros

$\mathrm{m} / \mathrm{s} n^{\circ} 6$, vol. 7 , juin-juillet 91 